\title{
Cystic Artery Pseudoaneurysm and Severe Cholangitis - A Rare Association - A Case Report
}

\section{Jaya Ghosh Chatterjee*, Sayan Das and Asokananda Konar}

Peerless Hospital and B K Roy Research Center, Kolkata, West Bengal, India

*Corresponding Author: Jaya Ghosh Chatterjee, Peerless Hospital and B K Roy Research Center, Kolkata, West Bengal, India.

Received: May 02, 2019; Published: November 11, 2019

DOI: $10.31080 /$ ASGIS.2019.02.0099

\begin{abstract}
Cystic artery pseudoaneurysm is a rare entity. It may be associated with severe cholecystitis, following laparoscopic cholecystectomy. Here we present a male patient with cystic artery pseudoaneurysm who presented with severe cholangitis. On evaluation he had cholangitic abscess, cholecystitis with underlying chronic pancreatitis with distal biliary stricture. He had haemobilia and underwent angioembolisation but later he required cholecystectomy due to acute cholecystitis - (gangrenous on preoperative findings).
\end{abstract}

Keywords: Cystic Artery Pseudoaneurysm; Cholangitis; MPD

\section{Introduction}

Cystic artery pseudoaneurysm is a rare entity. It is rarely seen following laparoscopic cholecystectomy and acute cholecystitis.

Here we present a case of an young male who had cholangitis with cholangitis abscess, incidentally found with cystic artery pseudoaneurysm and complications.

\section{Case}

34 years male, hypertensive, smoker and with moderate alcohol consumption, presented with pain upper abdomen of moderate intensity, persisting, for the last one month and increased over last 10 days. It was associated with nausea, but no vomiting, no clear relation to meal, or any radiation, referred pain. He noticed yellowish discolouration of urine followed by sclera over last 15 days and had high fever with chill for last 10 days. He had history of jaundice about 7 to 8 months back but it was not associated with fever, chill, pain abdomen and it subsided with conservative management at home. He also gave history of black stool intermittently for last 1 month. Initial management was done at his local place and was diagnosed as biliary pancreatitis and was referred to our hospital for further management. Outside investigations revealed Amylase and Lipase were raised but less than three times, LFT was deranged with raised bilirubin of 4.1/conjugated, raised SGOT, SGPT (>2-3 times), ALP was raised-330. MRCP outside showed dilated common Bile duct with filling defect-? Sludge/? stone, bulky pancreas, MPD mildly dilated and gallbladder distended with sludge.

He is married with one son. From a low middle class family, runs a small business. He was social drinker, smoker, hypertensive, non diabetic. He had no past history of blood transfusion or jaundice, but he had cerebrovascular accident with need for tracheostomy about an year ago, the details of which were unavailable. He was on amlodipine, but not on any antiplatelets or antiepileptics.

On admission- he was febrile with tachycardia (PR - 120/min), maintaining SPO2 98\% at room air, blood pressure of 110/60 mmHg. Abdomen was soft with right upper quadrant tenderness. Initial resuscitation was done with iv fluids, iv analgesia and iv antibiotics was started empirically suspecting cholangitis (piperacillin and tazobactam) and he was transferred to ITU from emergency. Laboratory Investigations was suggestive of cholangitis with total Bilirubin of 24.5/Conj 22.1, SGPT-73, SGOT-135, ALP-632, WBC total-24400, Neutrophil-92\%, Hb-9.8, creatinine-1.54, urea- 64, amylase-73(N), lipase-254(N), CRP-363(10). USG upper abdomen 
revealed dilated intra hepatic biliary radical, dilated CBD. CBD and GB loaded with sludge. MPD dilated with? double duct sign,? periampullary lesion. Repeat MRCP was done - Figure 1 (CT abdomen was avoided in view of raised creatinine) revealed significant dilated CBD, CHD, moderately dilated IHBR, sludge in CBD, abrupt narrowing at lower end of CBD around the head of the pancreas, significant hepatomegaly with multiple perihilar cholangitic abscess. Gall bladder grossly distended with pericholecystic inflammatory changes and multiple gall bladder calculi. There was a possibility of distal CBD stricture, bulky pancreas, and compression over MPD and prominence of distal MPD, minimal ascites. The final impressions was in favour of Cholangitis with cholangitic abscess, distal CBD stricture likely benign and GB calculi with cholecystitis.

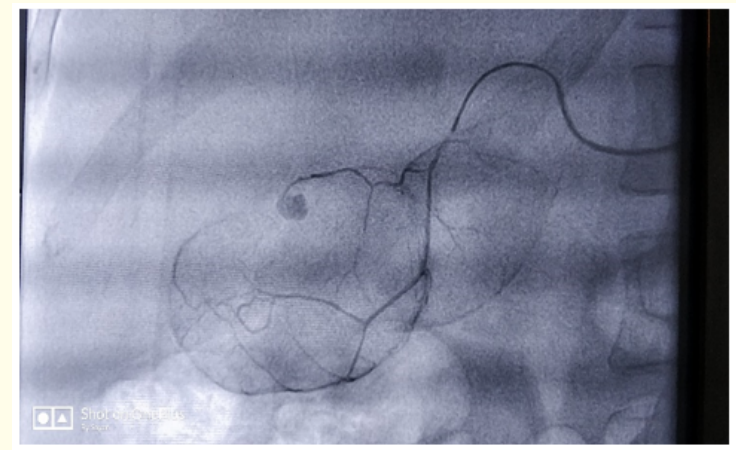

Figure 1: Cystic artery pseudoaneurysm.

ERCP was planned next day, however he had malena with drop of $2 \mathrm{gm} \mathrm{\%} \mathrm{in} \mathrm{haemoglobin.} \mathrm{So} \mathrm{an} \mathrm{urgent} \mathrm{UGI} \mathrm{scopey} \mathrm{was} \mathrm{done}$ which revealed blood mixed bile from the CBD -likely haemobilia. CT scan abdomen with mesenteric angiogram showed a brilliantly contrast enhancing lesion suggestive of pseudo aneurysm in the lateral wall of gall bladder supplied by post branch of cystic artery, large clot in GB lumen with dilated CBD and CHD. (His AKI was prerenal and was corrected by hydration). Micro coil embolization (Figure 2) was done on the same day and patient was haemodynamically stable. On the next day ERCP was performed with sphincterotomy blood mixed bile came out. There was difficulty in biliary cannulation, a stricture at lower CBD and few calculi were documented. Two pigtail stents were placed and normal bile flow was seen. Within 48 hours he had stable parameters. fever subsided and jaundice corrected rapidly $2.3 \mathrm{mg} / \mathrm{dl}$ from 24, TLC reduced to 14000 . He was advised for cholecystectomy , but he preferred to do it later. Hence we planned it on follow up after 3-4 weeks pre- ceded by detail re-evaluation of pancreatobiliary anatomy by EUS and stent removal following cholecystectomy.

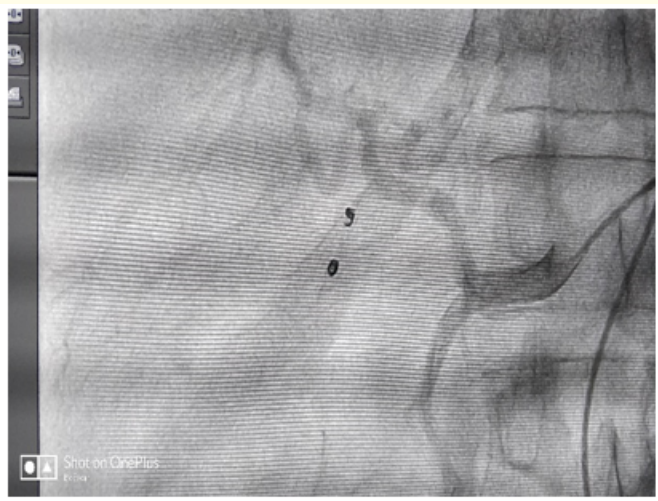

Figure 2: Coils in cystic artery.

Approximately after 9 days after coil embolization of pseudoaneurysm of cystic artery he developed an acute upper abdominal pain along with sweating and tachycardia. Clinically the gall bladder was palpable and tender. An USG showed acute cholecystitis and GB calculi. His TLC had increased to 39000 and blood CS showed E. Coli. antibiotics were changed to carbapenem. The surgeon was called and open cholecystectomy was performed on the next day. After 4 days post cholecystectomy he was discharged in haemodynamically stable condition with normal TLC-9000.

On FU after 1 month he was well, with normal CBC, TLC and LFT.

EUS examination revealed (Figure 3)pancreas has hypoechoic echotexture with atrophy of tail. Multiple hyperechoic and nonecho reflective foci, hyperechoic strands and lobules seen from neck to tail. The head is hetroechoic, lobulated forming an ill defined, mass (3 $\mathrm{cm}$ in diameter), encasing terminal CBD and MPD. No cyst or calculi seen. MPD was regular outline, with hyperechoic walls, mildly dilated ( $6 \mathrm{~mm}$ in neck just proximal to head mass, 2.5 $\mathrm{mm}$ in body).CBD dilated with sludge, stents in situ. MPV, SMV, SV were normal. EUS FNA obtained with 22 gauge needle, from head mass, copius haemorrhagic material obtained. Final impression was in favour of chronic pancreatitis with inflammatory head mass to rule out ca pancreas. FNA- cellular aspirate. no malignancy, likely inflammatory head mass. Repeat ERCP- Stent exchanged and now on stent exchange programme for last 8 months since admission. 


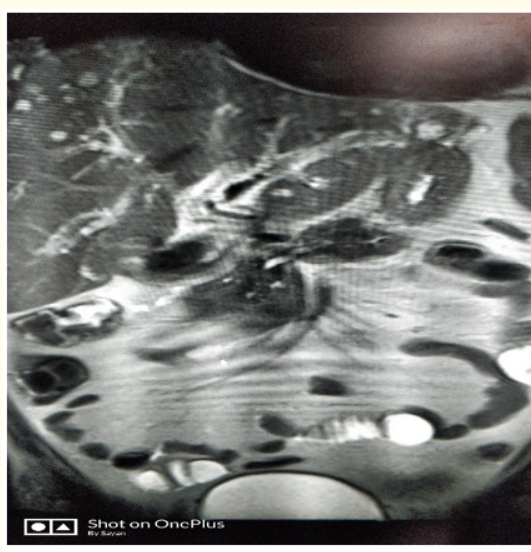

Figure 3: MRCP- multiple cholangitis abscess.

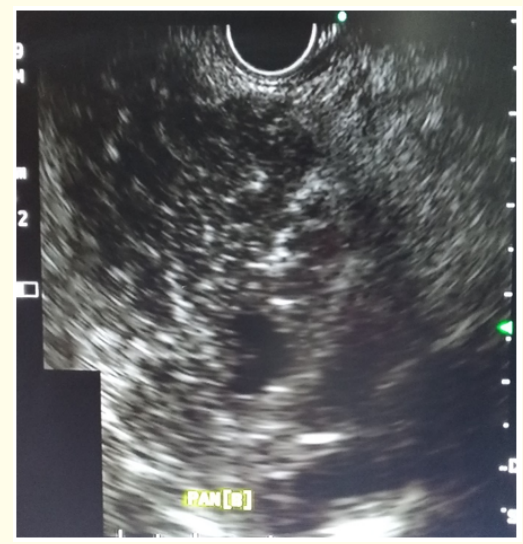

Figure 4: EUS- body of pancreas.

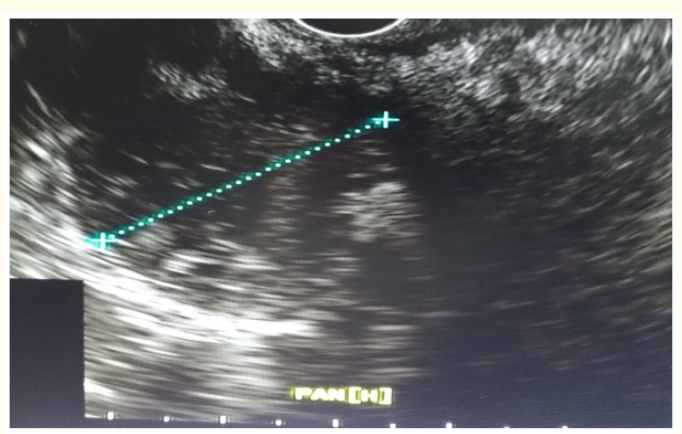

Figure 5: EUS- head of pancreas.

\section{Discussion}

Pseudoaneurysm of the cystic artery is a rare entity in itself. Majority of the cases are noticed after laparoscopic cholecystectomy and biliary procedures [1]. Acute cholecystitis is an uncommon cause of cystic artery pseudoaneurysms. There are few case reports on it. Mostly pseudoaneurysm of cystic artery presents with rupture, only 3 case reports of unruptured cystic artery pseudoaneurysm [2] have been reported. In ruptured pseudoaneurysm they present usually with haemobilia, pain right upper quadrant, and treatment of choice trans arterial coil embolization [3]. In case of pseudoaneurysm following acute cholecystitis (which is very rare), open cholecystectomy is preferred [3].

After coil embolization of cystic artery there may be some gall bladder ischaemia and subsequent need for cholecystectomy, but evidences are insufficient.

Here we present a case of a young male of cystic artery pseudoaneurysm, which was diagnosed on CT scan following haemobilia. Although he initially presented with cholangitis with cholangitic abcess, and with imaging evidence of cholecystitis(MRCP). Approximately 9 days after coil embolization he had acute cholecystitis which required cholecystectomy. It was unclear in our case whether cholecystitis is a cause or consequence of cystic artery Pseudoaneurysm and coil embolization. In view of rare presentation and rare association we like to share the case.

\section{Conclusion}

Cystic artery pseudoaneurysm us a rare presentation with haemobilia. Severe cholangitis and cholecystitis may be a cause for its development in our case. Chronic pancreatitis, with benign biliary stricture, cholangitis, cholecystitis may be all related to the development of pseudoaneurysm of cystic artery. Cholecystitis following cystic artery pseudoaneurysm angioembolisation may be an consequence due to ischaemia, although in our case there was evidence of cholecystitis before angioembolisation.

\section{Bibliography}

1. Nkwam Nkwam. "Unruptured Pseudoaneurysm of the cystic artery associated with acute calculus cholecystiytis". Journal of Surgical Case Report (2010): 4. 
2. OL De Molk., et al. "Pseudoaneurysm of Cystic artery afyer laparoscopic cholecystectomy”. HPB(Oxford) 8 (2006): 318-319.

3. Deniz Alis., et al. "Laparoscopic management of a very rare case; Cystic artery pseudoanerysm secondary to acute cholecystitis". Case Reports in Surgery (2016).

Volume 2 Issue 9 November 2019

(C) All rights are reserved by Jaya Ghosh Chatterjee., et al. 